\title{
Simplex case
}

National Cancer Institute ( $\mathrm{NCl})$

\section{Source}

National Cancer Institute (NCI). Simplex case.

A single, isolated occurrence of an inherited condition in a family. Simplex cases can result from certain inheritance patterns or be caused by de novo mutations or reduced penetrance within the family. It can also result from nongenetic causes such as adoption or alternate paternity. 